\title{
Prevalência de doença hepática não-alcoólica em crianças e adolescentes obesos: uma revisão sistemática
}

\author{
Prevalence of nonalcoholic fatty liver disease in obese children and adolescents: a systematic review
}

Patricia de Carvalho Padilha1, Hélio Fernandes da Rocha ${ }^{2}$, Naylor Alves ${ }^{3}$, Wilza Arantes F. Peres ${ }^{4}$

\section{RESUMO}

Objetivo: Revisar publicações que investigaram a prevalência de doença hepática gordurosa não-alcoólica (DHGNA) em crianças.

Fontes de dados: Bancos de dados Medline (versão PubMed) e Latin American and Caribbean Centre on Health Sciences Information (LILACS), considerando-se o período de 1997 a 2008. Aplicou-se a combinação dos seguintes descritores: "nonalcobolic fatty liver disease", "hepatic steatosis", "nonalcoholic steatobepatitis", "overweight children", "overweight adolescent", "pediatric obesity", "children obesity", "childhood obesity".

Síntese dos dados: Foram selecionados 14 artigos: cinco transversais, um caso-controle e oito de coorte. A concordância entre os avaliadores na classificação da qualidade dos artigos foi considerada ótima $(k=0,81)$, com intervalo de confiança de $95 \%(0,52-1,00 ; p<0,001)$; porém, apenas um estudo foi considerado de excelente qualidade. Todos os estudos demonstraram associação entre obesidade e DHGNA, com prevalências variando de 3,0 a $60,3 \%$. Os artigos analisados indicam que as alterações bioquímicas e de imagem são rapidamente normalizadas com a programação da perda ponderal por meio de dieta e exercício físico. Observam-se prevalências maiores em meninos, com o aumento da idade. Uma associação relatada com frequência foi a da resistência insulínica com a progressão da DHGNA.
Conclusões: São necessários estudos cuidadosamente desenhados para esclarecer a relação entre obesidade e DHGNA na infância. Recomenda-se que todas as crianças obesas sejam rastreadas, já que a DHGNA representa a maior causa de agravo hepático na infância.

Palavras-chave: Doença hepática gordurosa não alcoólica; obesidade; criança.

\section{ABSTRACT}

Objective: To review studies that investigated the prevalence of nonalcoholic fatty liver disease (NAFLD) in children.

Data Source: Medline (PubMed) and Latin American and Caribbean Centre on Health Sciences Information (LILACS) were searched regarding the period from 1997 to 2008, using the combination of the following descriptors: "nonalcoholic fatty liver disease", "hepatic steatosis", "nonalcoholic steatohepatitis", "overweight children”, "overweight adolescent”, "pediatric obesity", "children obesity", "childhood obesity".

Data synthesis: 14 manuscripts were selected: five cross-sectional, one case-control, and eight cohort studies. Evaluation of quality between reviewers showed optimal agreement $(k=0.81)$, with a $95 \%$ confidence interval of $0.52-1.00(p<0.001)$, but only one study was considered excellent. All studies showed association between obesity and NAFLD, with a prevalence rate varying from 3.0
Instituição: Universidade Federal do Rio de Janeiro (UFRJ), Rio de Janeiro, RJ, Brasil

'Doutoranda em Ciências Nutricionais do Programa de Pós-Graduação em Nutrição do Instituto de Nutrição Josué de Castro da UFRJ; Nutricionista do Instituto de Puericultura e Pediatria Martagão Gesteira da UFRJ, Rio de Janeiro, RJ, Brasil

${ }^{2}$ Mestre em Medicina pela Faculdade de Medicina da UFRJ; Professor Assistente de Pediatria da Faculdade de Medicina da UFRJ, Rio de Janeiro, RJ, Brasil

${ }^{3}$ Doutor em Medicina pela Faculdade de Medicina da UFRJ; Professor Adjunto de Pediatria da Faculdade de Medicina da UFRJ, Rio de Janeiro, RJ, Brasil ${ }^{4}$ Doutora em Clínica Médica pela UFRJ; Professora Adjunta do Departamento de Nutrição e Dietética do Instituto de Nutrição da UFRJ, Rio de Janeiro, RJ, Brasil
Endereço para correspondência:

Patricia de Carvalho Padilha

Rua Gramado, 475, bloco C - Campo Grande

CEP 23050-090 - Rio de Janeiro/RJ

E-mail: paticpadilha@yahoo.com.br

Conflitos de interesse: nada a declarar

Recebido em: 19/3/2009

Aprovado em: 26/7/2009 
to $60.3 \%$. The studies indicate that the biochemical and image alterations normalize with programs of weight loss that apply diets and physical exercises. There was a high prevalence of NAFLD among boys, increasing with age. Insulin resistance was often reported as associated with the progression of NAFLD.

Conclusions: Carefully designed studies are needed to clarify the association between obesity and NAFLD during childhood. NAFLD, which is the main cause of infantile liver disorder, should be investigated in all obese children.

Key-words: Nonalcoholic fatty liver disease; obesity; children.

\section{Introdução}

Ao longo das últimas décadas, a prevalência de obesidade infantil tem aumentado de forma significativa, sendo considerada um dos principais problemas de saúde pública e uma epidemia global $^{(1)}$. Dados do National Health and Nutrition Examination Survey (NHANES) ${ }^{(2)}$, de 1991 a 2002, mostraram que $10 \%$ das crianças entre dois e cinco anos e $16 \%$ das crianças e adolescentes entre seis e 19 anos estavam obesas. O Brasil segue a tendência mundial e já apresenta alta prevalência de sobrepeso e obesidade em sua população jovem, pois, em apenas 30 anos, o número de crianças e adolescentes com sobrepeso ou obesas subiu de 4 para $18 \%$ e de 7,5 para $15,5 \%$ em meninos e meninas, respectivamente $\mathrm{e}^{(3)}$.

Muitos estudos apontam o aumento da prevalência de obesidade infantil como um importante preditor de obesidade na vida adulta e de várias comorbidades amplamente estabelecidas na literatura, como dislipidemias, inflamações crônicas, resistência à insulina, diabetes tipo 2, hipertensão e complicações ortopédicas, entre outras. Nesse sentido, outro aspecto descrito com frequência na literatura é o aumento da incidência de doença hepática gordurosa não-alcoólica (DHGNA). Trata-se de problema clínico emergente em obesos, mesmo em crianças e adolescentes, podendo levar à cirrose hepática $^{(1,3-5)}$.

A DHGNA é caracterizada pelo acúmulo de gordura no fígado (esteatose) não relacionado ao uso de álcool. A esteato-hepatite não-alcoólica (EHNA) é uma DHGNA na qual a presença da gordura é desencadeada por um processo inflamatório no fígado (hepatite). Assim, a esteatose hepática ("fígado gorduroso") e a EHNA são apresentações diferentes da DHGNA, sendo que a primeira pode evoluir para a segunda e, posteriormente, para cirrose, que é resultado de uma inflamação crônica e irreversível ${ }^{(5)}$. Tominaga et a $l^{(6)}$ verificaram que $20 \%$ das crianças ou adolescentes obesos com esteatose hepática desenvolveram cirrose ou carcinoma hepatocelular. A alta adiposidade visceral pode acentuar o grau da lesão hepática ${ }^{(1)}$.

A real prevalência de DHGNA na população pediátrica é desconhecida em função da falta de informações. Os dados disponíveis sugerem que a esteatose hepática afeta 2,6\% das crianças e 9,6\% dos adolescentes; entretanto, sua prevalência varia de 12 a $80 \%$ em crianças obesas ${ }^{(1,5)}$. Todos os pacientes são encorajados a reduzir a ingestão de gorduras e realizar exercícios físicos regulares. Ainda não existe tratamento farmacológico específico para o controle da esteatose hepática em crianças $^{(4,5)}$. No que se refere à DHGNA, especial atenção precisa ser creditada à identificação dos casos, uma vez que esta é provavelmente a causa mais comum de doença hepática nos grupos de escolares e adolescentes ${ }^{(1,5,7)}$.

Considerando-se a relevância deste tema e a inexistência de um consenso na literatura, os objetivos desta revisão foram identificar a prevalência de DHGNA em crianças obesas, embasando a discussão sobre a necessidade de elaborar modelos de intervenção nutricional direcionados às crianças com obesidade e valorizar outras complicações, como a esteatose hepática, além daquelas classicamente descritas no estudo da obesidade infantil.

\section{Método}

Trata-se de estudo de revisão sistemática da literatura com seleção de artigos a partir de estratégia de busca definida e passível de reprodução sobre a prevalência mundial da DHGNA em crianças e adolescentes obesos.

A revisão foi realizada seguindo-se a etapas de colaboração da biblioteca Cochrane ${ }^{(8)}$. Os tipos de estudos considerados na inclusão foram transversais, caso-controle e longitudinais. Estudos de caso, estudos não controlados, estudos com animais, in vitro, de revisão, estudos não conclusivos e com metodologia não definida foram excluídos da análise. Para a inclusão, os estudos deveriam contar com a participação de crianças e adolescentes de ambos os sexos com DHGNA, identificada por meio de biópsia hepática e/ou ultrassonografia (USG) e/ou ressonância magnética e/ou alteração dos níveis de alanina aminotransferase (ALT). Artigos com população adulta foram excluídos. A variável desfecho foi a prevalência de crianças e adolescentes com DHGNA. 
Os artigos foram identificados por meio de busca nas bases de dados eletrônicas Medline (Versão PubMed) e Latin American and Caribbean Centre on Health Sciences Information (LILACS), considerando-se o período de 1997 a 2008, restringindo-se aos idiomas inglês, português e espanhol. Os descritores utilizados na busca foram: "nonalcoholic fatty liver disease OR hepatic steatosis OR nonalcoholic steatohepatitis AND overweight children OR overweight adolescent OR pediatric obesity OR children obesity OR childhood obesity".

Os resultados das buscas foram rastreados independentemente por dois nutricionistas qualificados, a partir dos títulos e resumos dos artigos. Identificados os estudos relevantes, a publicação completa foi adquirida e revisada pelos dois profissionais para determinar a elegibilidade para inclusão final. Os dois revisores selecionaram artigos independentemente, de acordo com critérios de seleção pré-especificados. A concordância das escolhas foi medida pelo cálculo do coeficiente Kappa tradicional e seu intervalo de confiança de $95 \%$ ( $k$, IC95\%). Discordâncias foram resolvidas por consenso e, quando este não pôde ser alcançado, foi definido por um terceiro julgador.

A qualidade metodológica dos artigos incluídos foi avaliada de forma independente pelos dois revisores/autores, por meio das recomendações do Strengthening the Reporting of Observational Studies in Epidemiology Statement (STROBE) ${ }^{(9)}$, com especial consideração sobre viés de seleção, viés de detecção e perda de seguimento. Três categorias para avaliação da qualidade foram estabelecidas: $\mathrm{A}$ - quando o estudo preenchia mais de $80 \%$ dos critérios estabelecidos no STROBE; B - quando 50-80\% dos critérios do STROBE eram preenchidos; $\mathrm{C}$ - quando menos de $50 \%$ dos critérios foram preenchidos. A concordância entre os avaliadores foi novamente medida para a escala de qualidade por meio do cálculo do coeficiente Kappa e as divergências foram resolvidas por consenso.

As informações foram analisadas utilizando-se o pacote estatístico Statistical Package for the Social Sciences (SPSS), versão 13.0. Os dados foram apresentados em percentuais, de acordo com as prevalências descritas. A concordância medida pelo Kappa seguiu a orientação da literatura: kappa $<0,10$ ausência de concordância, $<0,40$ - concordância fraca, 0,400,75 - concordância boa e $>0,75$ - concordância excelente ${ }^{(10)}$.

\section{Resultados}

A pesquisa inicial realizada nas bases de dados eletrônicas identificou 149 referências. Após análise criteriosa dos artigos identificados, exclusão dos repetidos e a leitura dos resumos, realizou-se nova seleção, que totalizou 25 trabalhos, nos quais a faixa etária variou de pré-escolar a adolescência. Posteriormente à leitura dos artigos selecionados na íntegra, oito foram excluídos com base nos critérios de inclusão e dois não foram localizados. A Figura 1 apresenta o fluxograma de seleção da amostra final.

Dos 14 artigos selecionados, cinco foram estudos transversais, um foi caso-controle e oito, de coorte. Em relação ao local, quatro foram realizados nos Estados Unidos, cinco na Itália, um na Alemanha, um em Israel, dois no Brasil e um na China.

Nesta análise, todos os estudos demonstraram associação entre obesidade e DHGNA, com prevalências variando de 3,0 a $60,3 \%$. Apenas um dos artigos utilizou o indicador dietético da avaliação nutricional ${ }^{(11)}$.

Os estudos foram consensuais ao considerarem o excesso de peso como um fator independente para o aumento do risco de fibrose hepática. A maioria dos estudos descreveu prevalências significativamente maiores em meninos, com o aumento da idade, e, dentre as diferentes raças e etnias, os hispânicos destacaram-se por apresentarem maiores prevalências.

Dentre os autores, Quirós-Tejeira $e t$ a $l^{(11)}$ e Schwimmer et $a^{l^{(12)}}$ defendem que as alterações bioquímicas e de imagem são rapidamente normalizadas com a programação da perda de peso por meio de dieta e exercício físico.

Uma associação relatada com frequência foi a resistência insulínica, possivelmente associada à progressão da DHGNA. De acordo com as evidências apresentadas, pareceu haver

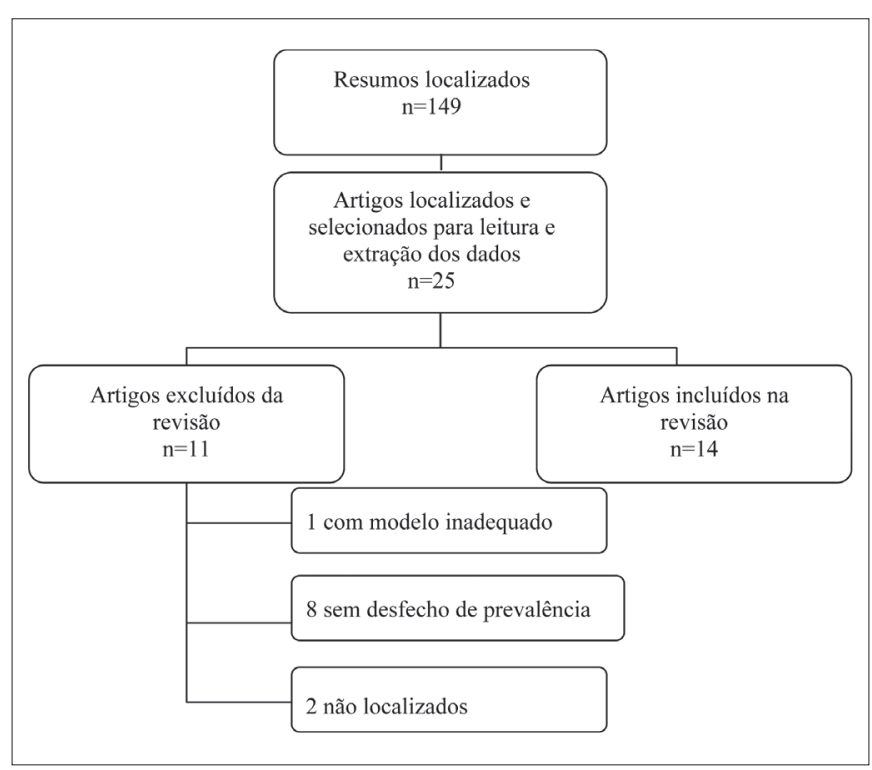

Figura 1 - Etapas de inclusão e exclusão de estudos. 
tendência de novos estudos que relacionassem os níveis de adiponectina e resistina, pois a DHGNA está inversamente relacionada aos valores dessas adipocitocinas envolvidas no metabolismo de carboidratos e lipídios.

Estimou-se o coeficiente de Kappa, levando-se em conta a avaliação dos dois profissionais nutricionistas. A seleção independente dos artigos para inclusão nesta revisão demonstrou excelente concordância entre as escolhas dos avaliadores $(k=1,00$; IC95\% 0,71-1,00; $p<0,001)$. A avaliação da concordância entre os avaliadores na classificação da qualidade dos artigos demonstrou ótima concordância ( $k=0,81$; IC95\% $0,52-1,00 ; p<0,001)$. Do total de estudos analisados, apenas um foi considerado de qualidade A $(7,1 \%) ; 11(78,6 \%)$ e dois $(14,3 \%)$ mostravam qualidade $\mathrm{B}$ e $\mathrm{C}$, respectivamente. Informações sobre as perdas de seguimento foram obtidas em apenas um dos estudos ${ }^{(11)}$, embora não quantificadas.

As Tabelas 1 e 2 trazem os dados dos 14 estudos $^{(11-24)}$ referentes à prevalência de DHGNA, área estudada, tipo de estudo, tamanho amostral e o método diagnóstico utilizado.

\section{Discussão}

Embora a DHGNA seja uma complicação pouco relatada na faixa etária pediátrica, os resultados desta revisão

Tabela 1 - Características dos estudos selecionados quanto a desenho, local e ano de publicação

\begin{tabular}{|c|c|c|c|}
\hline Autor & Tipo de estudo & Local do estudo & Ano \\
\hline Franzese et $a^{(13)}$ & Coorte & Itália & 1997 \\
\hline Zou et a/(14) & Caso-controle & China & 2005 \\
\hline Schwimmer et al(12) & Coorte & Estados Unidos & 2005 \\
\hline Dâmaso et $a^{(15)}$ & Coorte & Brasil & 2006 \\
\hline Nobili et $a^{(16)}$ & Coorte & Itália & 2006 \\
\hline Radetti et a/(17) & Transversal & Itália & 2006 \\
\hline Schwimmer et al(18) & Transversal & Estados Unidos & 2006 \\
\hline Pacifico et a/(19) & Transversal & Itália & 2007 \\
\hline Burgert et $a^{(20)}$ & Coorte & Estados Unidos & 2006 \\
\hline Perseghin et $a /^{(21)}$ & Coorte & Itália & 2006 \\
\hline Sagi et a/(22) & Coorte & Israel & 2007 \\
\hline Quirós-Tejeira et a/(11) & Coorte & Estados Unidos & 2007 \\
\hline Imhof et $a^{(23)}$ & Transversal & Alemanha & 2007 \\
\hline Souza et $a^{(24)}$ & Transversal & Brasil & 2008 \\
\hline
\end{tabular}

Tabela 2 - Características dos estudos selecionados quanto a tamanho amostral, prevalência e método diagnóstico da DHGNA em crianças e adolescentes obesos

\begin{tabular}{|c|c|c|c|}
\hline Autor & Amostra (n) & Prevalência (\%) & Métodos diagnósticos \\
\hline Franzese et a/(13) & 75 & $\begin{array}{l}53,0 \\
25,0\end{array}$ & $\begin{array}{c}\text { Ultrassonografia e } \\
\text { Alteração dos níveis de ALT }\end{array}$ \\
\hline Zou et a/(14) & 113 & 55,8 & Alteração dos níveis de ALT \\
\hline Schwimmer et al/12) & 127 & 23,0 & Alteração dos níveis de ALT \\
\hline Dâmaso et a/(15) & 28 & 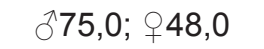 & Ultrassonografia \\
\hline Nobili et $a)^{(16)}$ & 84 & 58,1 & Biópsia hepática \\
\hline Radetti et $a /^{(17)}$ & 44 & 31,8 & Ressonância magnética \\
\hline Schwimmer et a/(18) & 742 & 13,0 & Biópsia hepática \\
\hline Pacifico et a/ ${ }^{(19)}$ & 50 & 40,0 & Ressonância magnética \\
\hline Burgert et $a^{(20)}$ & 392 & 14,0 & Alteração dos níveis de ALT \\
\hline Perseghin et $a /^{(21)}$ & 54 & 30,0 & Biópsia hepática \\
\hline Sagi et a/(22) & 58 & 60,3 & Ultrassonografia \\
\hline Quiróz-Tejeira et a/(11) & 517 & 24,0 & Alteração dos níveis de ALT \\
\hline Imhof et $a^{(23)}$ & 372 & 13,7 & Ultrassonografia \\
\hline Souza et $a^{(24)}$ & 67 & $\begin{array}{c}57,4 \\
3,0\end{array}$ & $\begin{array}{c}\text { Ultrassonografia e } \\
\text { Alteração dos níveis de ALT }\end{array}$ \\
\hline
\end{tabular}

ALT: alanina aminotransferase. DHGNA: doença hepática gordurosa não alcoólica 
sistemática apontam que os dados podem estar subestimados, em especial entre crianças obesas, tendo em vista as altas taxas descritas. Certamente, a real prevalência de DHGNA ainda é desconhecida, mas os presentes achados confirmam a necessidade de estudos de base populacional com métodos diagnósticos viáveis, que estimulem a triagem dessa complicação na rotina de acompanhamento da criança obesa, uma vez que a obesidade é o maior fator de risco associado à DHGNA ${ }^{(5,7,25)}$.

No entanto, são escassos os estudos prospectivos que tenham descrito a associação entre obesidade infantil e DHGNA e a patogênese da alteração hepática. Os estudos que investigaram a associação entre essas variáveis são contraditórios. Algumas inconsistências nessa associação podem ser atribuídas em parte à seleção inadequada do tipo de estudo, à não-inclusão de grupo de comparação, à falta de análise das taxas de perda de seguimento, à ausência de controle para variáveis de confusão, à falta de avaliação da homogeneidade entre os grupos analisados e às diferenças no critério diagnóstico de DHGNA.

Uma das razões do interesse em se estudar a associação entre obesidade e DHGNA decorre da relevante prevalência de ambos os desfechos e da necessidade de que potenciais fatores determinantes possam ser controlados, diminuindo, assim, a frequência desses eventos e suas complicações. As taxas de DHGNA ainda não são conhecidas na maioria dos países da América Latina, onde as prevalências de obesidade crescem assustadoramente em função da transição epidemiológica ${ }^{(2,26)}$, devendo ser uma preocupação dos comitês nacionais e internacionais de saúde.

Os estudos brasileiros sobre o assunto foram bem conduzidos e ressaltaram a importância da ampliação dos conhecimentos em relação à DHGNA e outros distúrbios nutricionais, além da obesidade e, especialmente, o impacto da associação desses distúrbios na potencialização de efeitos, favorecendo sua ocorrência ${ }^{(13,24)}$.

A idade mostrou-se relevante nos estudos avaliados. Os estudos analisados incluíram adolescentes ( $<18$ anos) e/ou pré-escolares a partir de dois anos. Schwimmer $e t a^{(18)}$ demonstraram que a prevalência varia com o aumento da idade, sendo $0,7 \%$ na faixa etária de dois a quatro anos, e, nas faixas de cinco a nove e 15-19 anos, 3,3 e 17,3\%, respectivamente. Com esses dados, os autores sugerem que a faixa de cinco a nove anos seria a ideal para direcionar as ações de prevenção da DHGNA.

Alguns estudos apontaram que os meninos possuem maiores riscos de apresentar DHGNA. Dentre eles, Schwimmer $e t$ $a^{(12)}$ defendem que, em trabalhos com crianças, os meninos geralmente apresentam prevalências de DHGNA na razão $2: 1$, quando comparados às meninas. Tais achados corroboram os encontrados com a população adulta, na qual a razão é de 1,5:1. Dentre as possíveis explicações para maiores taxas de gordura hepática em indivíduos do sexo masculino destacase a maior propensão desse grupo de acumular o excesso de gordura corporal no compartimento intra-abdominal e a influência dos hormônios sexuais. É fato que essas hipóteses ainda não foram testadas em crianças.

A maior suscetibilidade dos hispânicos para desenvolverem a alteração hepática aqui estudada é também descrita em outros trabalhos ${ }^{(5,27)}$. A importância desses achados decorre das evidências de que os adultos hispânicos têm uma alta taxa de morbimortalidade relacionada às causas hepáticas. Além disso, crianças e adolescentes hispânicos parecem ser mais propensos à fibrose hepática avançada ${ }^{(28)}$.

Sabe-se que o aumento na prevalência de DHGNA pode ser decorrente de várias alterações no metabolismo, destacando-se a captação, síntese, degradação ou secreção de lipídios, resultantes da resistência insulínica ${ }^{(25,29)}$. Frente a esse quadro de resistência à ação da insulina, a lipólise encontra-se aumentada no adipócito, resultando em aumento na liberação de ácidos graxos livres, os quais serão captados pelos hepatócitos, onde ocorrerá lipogênese ${ }^{(29)}$. Isso explica parcialmente a relação da resistência insulínica no desenvolvimento da DHGNA, amplamente mencionada nos trabalhos.

Quanto à importância do rastreamento da DHGNA em crianças obesas, os estudos são unânimes ao reforçarem sua necessidade, porém ainda são confusos quanto ao posicionamento acerca dos critérios diagnósticos. Sagi et al ${ }^{(22)}$ defendem que somente a mensuração das enzimas hepáticas é insuficiente para o diagnóstico precoce, sendo necessária a realização da USG. Souza et al ${ }^{(24)}$ concluíram que o percentual de inadequação das enzimas hepáticas (ALT) é baixo e o de alterações ultrassonográficas é alto, corroborando os demais achados. Essas diferenças no critério diagnóstico devem ser responsáveis pela ampla variação de frequência de esteatose obtida em diferentes estudos.

Embora ainda não exista um consenso sobre o tratamento farmacológico específico para o controle da DHGNA, todos os pacientes são encorajados a reduzir a ingestão de gorduras e realizar exercícios físicos regulares, com o objetivo de aumentar o gasto energético diário ${ }^{(5,29)}$. A falta de um modelo de intervenção direcionado a crianças com esteatose hepática é notória e diversas experiências isoladas vêm sendo testadas, dentre elas, a avaliação da perda ponderal na regressão da 
lesão hepática, o uso de agentes farmacológicos, a utilização de antioxidantes e a indicação de exercícios físicos ${ }^{(5)}$.

Manton et a ${ }^{(29)}$ defendem a hipótese de que crianças obesas tendem a apresentar concentrações reduzidas de antioxidantes lipossolúveis. Assim, o estresse oxidativo pode favorecer a patogênese da DHGNA, resultando em síntese no tecido adiposo do fator de necrose tumoral (TNF). O TNF é antagonista de receptores de insulina, levando à intolerância a glicose, dislipidemia e esteatose hepática. Ademais, o aumento da peroxidação lipídica pode levar ao aumento da ativação das células hepáticas estreladas, sendo esta a etapa inicial da fibrose hepática. Contudo, nenhum dos estudos analisados, incluindo os de intervenção, mencionou o papel dos antioxidantes no tratamento da DHGNA.

Segundo Scheen e Luyckx ${ }^{(30)}$, a redução de massa adiposa diminui o grau de lesão hepática e suas consequências. Porém, a redução abrupta de massa corporal pode promover alterações hepáticas indesejáveis, como toxicidade, agravando o processo necroinflamatório ${ }^{(29)}$. Finalmente, Bouneva e Kirby ${ }^{(31)}$ mencionaram que não é necessário realizar exercícios de alta intensidade para prevenir e controlar a doença. Desta forma, tratamentos multidisciplinares para mudanças no estilo de vida são desejáveis. A afirmação acima sustenta os achados referentes aos estudos de Franzese et a ${ }^{(13)}$ e Nobili et $a l^{(16)}$, presentes nesta casuística, que defendem a mudança no estilo de vida como uma estratégia de redução dos agravos decorrentes da DHGNA.

Dâmaso $e t$ al ${ }^{(15)}$ sugerem que o tratamento multidisciplinar, com intervenção nutricional e atividade física, foi eficaz para reduzir a adiposidade visceral, contribuindo para

\section{Referências bibliográficas}

1. Mathur P, Das MK, Arora NK. Non-alcoholic fatty liver disease and childhood obesity. Indian J Pediatr 2007;74:401-7.

2. Centers for Disease Control and Prevention (CDC) [homepage on the Internet] [cited 2007 Jun 16]. Available from: http://www.cdc.gov

3. Instituto Brasileiro de Geografia e Estatística (IBGE) [homepage on the Internet]. Diretoria de Pesquisas, Coordenação de Índices de preços, Pesquisa de Orçamentos familiares, 2002-2003 [cited 2007 Dec 10]. Available from: http://www.ibge.gov.br/home/estatistica/populacao/condicaodevida/pof/2002/ pof2002.pdf

4. Schwimmer JB. Definitive diagnosis and assessment of risk for nonalcoholic fatty liver disease in children and adolescents. Semin Liver Dis 2007;27:312-8.

5. Papandreou D, Rousso I, Mavromichalis I. Update on non-alcoholic fatty liver disease in children. Clin Nutr 2007;26:409-15.

6. Tominaga K, Kurata JH, Chen YK, Fujimoto E, Miyagawa S, Abe I et al. Prevalence of fatty liver in Japanese children and relationship to obesity. An epidemiological ultrasonographic survey. Dig Dis Sci 1995;40: 2002-9 reduzir a prevalência de esteatose hepática não-alcoólica em adolescentes obesos.

Entretanto, questionamentos emergem sobre a efetividade dessas medidas de intervenção, assim como a padronização por meio de protocolos para manejo das intercorrências hepáticas na criança obesa, valorizando essa complicação que pode progredir para estágios mais avançados da doença hepática, como a cirrose e o carcinoma hepatocelular, com desfechos possivelmente letais.

\section{Conclusões}

A realização de estudos cuidadosamente desenhados, com recrutamento de indivíduos com características similares e utilização de critérios diagnósticos uniformes, poderá contribuir para produzir novas evidências acerca da relação entre obesidade e DHGNA. Tais evidências podem ser importantes para subsidiar o profissional de saúde na aplicação preventiva de uma abordagem adequada ao enfrentamento do problema.

Recomenda-se que todas as crianças obesas sejam rastreadas na prática clínica, levando-se em consideração múltiplos indicadores da avaliação nutricional. Ressalta-se a necessidade de valorizar outras complicações além das cardiovasculares, uma vez que a DHGNA representa a maior causa de agravo hepático na infância. Por fim, a presente revisão poderá contribuir para elaborar modelos de intervenção nutricional aplicados a crianças com obesidade e suas complicações hepáticas.
7. Cavanilles Walker E, Solar Boga A, García Alonso L, Lorenzo Patiño MJ. Effectiveness of weight loss in the treatment of non-alcoholic steatohepatitis in an obese adolescent. An Pediatr (Barc) 2007; 66:184-7.

8. The Cochrane Colaboration [homepage on the Internet]. What are systematic reviews and protocols? [cited 2008 Jul 11]. Available from: http://www. thecochranelibrary.com/view/0/AboutCochraneSystematicReviews.html

9. STROBE (Strengthening the Reporting of Observational Studies in Epidemiology Statement) [homepage on the Internet]. Checklist of items that should be included in reports of observational studies [cited 2008 Jul 19]. Available from: http://www.neurology.org/misc/strobechklist.pdf

10. Hosmer DW, Lemeshow S. Applied logistic regression. New York: Wiley; 1989.

11. Quirós-Tejeira RE, Rivera CA, Ziba TT, Mehta N, Smith CW, Butte NF. Risk for nonalcoholic fatty liver disease in Hispanic youth with $\mathrm{BMI} \geq$ or $=95$ th percentile. J Pediatr Gastroenterol Nutr 2007;44:228-36.

12. Schwimmer JB, McGreal N, Deutsch R, Finegold MJ, Lavine JE. Influence of gender, race, and ethnicity on suspected fatty liver in obese adolescents. Pediatrics 2005;115:e561-5. 
13. Franzese A, Vajro P, Argenziano A, Puzziello A, lannucci MP, Saviano MC et al. Liver involvement in obese children. Ultrasonography and liver enzyme levels at diagnosis and during follow-up in an Italian population. Dig Dis Sci 1997;42:1428-32.

14. Zou CC, Liang L, Hong F, Fu JF, Zhao ZY. Serum adiponectin, resistin levels and non-alcoholic fatty liver disease in obese children. Endocr J 2005;52:519-24.

15. Dâmaso AR, Tock L, Tufik S, Prado WL, Stella SG, Fisberg M et al. Tratamento multidisciplinar reduz o tecido adiposo visceral, leptina, grelina e a prevalência de esteatose hepática não alcoólica (NAFLD) em adolescentes obesos. Rev Bras Med Esporte 2006;12:263-7.

16. Nobili V, Marcellini M, Devito R, Ciampalini P, Piemonte F, Comparcola D et al. NAFLD in children: a prospective clinical-pathological study and effect of lifestyle advice. Hepatology 2006;44:458-65.

17. Radetti G, Kleon W, Stuefer J, Pittschieler K. Non-alcoholic fatty liver disease in obese children evaluated by magnetic resonance imaging. Acta Paediatr 2006;95:833-7.

18. Schwimmer JB, Deutsch R, Kahen T, Lavine JE, Stanley C, Behling C. Prevalence of fatty liver in children and adolescents. Pediatrics 2006;118:1388-93.

19. Pacifico L, Celestre M, Anania C, Paoloantonio P, Chiesa C, Laghi A. MRI and ultrasound for hepatic fat quantification: relationships to clinical and metabolic characteristics of pediatric nonalcoholic fatty liver disease. Acta Paediatr 2007;96:542-7.

20. Burgert TS, Taksali SE, Dziura J, Goodman TR, Yeckel CW, Papademetris $X$ et al. Alanine aminotransferase levels and fatty liver in childhood obesity: associations with insulin resistance, adiponectin, and visceral fat. J Clin Endocrinol Metab 2006;91:4287-94.

21. Perseghin G, Bonfanti R, Magni S, Lattuada G, De Cobelli F, Canu T et al. Insulin resistance and whole body energy homeostasis in obese adolescents with fatty liver disease. Am J Physiol Endocrinol Metab 2006; 291:697-703.

22. Sagi R, Reif S, Neuman G, Webb M, Phillip M, Shalitin S. Nonalcoholic fatty liver disease in overweight children and adolescents. Acta Paediatr 2007;96:1209-13.

23. Imhof A, Kratzer W, Boehm B, Meitinger K, Trischler G, Steinbach G et al. Prevalence of non-alcoholic fatty liver and characteristics in overweight adolescents in the general population. Eur J Epidemiol 2007;22:889-97.

24. Souza FI, Amâncio OM, Sarni RO, Pitta TS, Fernandes AP, Fonseca FL et al. Nonalcoholic fatty liver disease in obese children. Rev Paul Pediatr 2008;26:136-41.

25. Schwimmer JB, Deutsch R, Rauch JB, Behling C, Newbury R, Lavine JE. Obesity, insulin resistance, and other clinicopathological correlates of pediatric nonalcoholic fatty liver disease. J Pediatr 2003;143:500-5.

26. Monteiro CA, Conde WL. Tendência secular da desnutrição e da obesidade na infância na cidade de São Paulo (1974-1996). Rev Saúde Pública 2000;34 (6 Supl):52-61.

27. Singh GK, Hoyert DL. Social epidemiology of chronic liver disease and cirrhosis mortality in the United States, 1935-1997: trends and diferentials by ethnicity, socioeconomic status, and alcohol consumption. Hum Biol 2000;72:801-20.

28. Browning JD, Kumar KS, Saboorian MH, Thiele DL. Ethnic differences in the prevalence of cryptogenic cirrhosis. Am J Gastroenterol 2004;99:292-8.

29. Manton ND, Lipsett J, Moore DJ, Davidson GP, Bourne AJ, Couper RT. Non-alcoholic steatohepatitis in children and adolescents. Med J Aust 2000;173:476-9.

30. Scheen AJ, Luyckx FH. Obesity and liver disease. Best Pract Res Clin Endocrinol Metab 2002;16:703-16.

31. Bouneva I, Kirby DF. Management of nonalcoholic fatty liver disease: weight control. Clin Liver Dis 2004;8:693-713. 